\title{
Tratamento da hipertensão resistente: um estudo randomizado de clonidina versus espironolactona. Estudo ReHOT
}

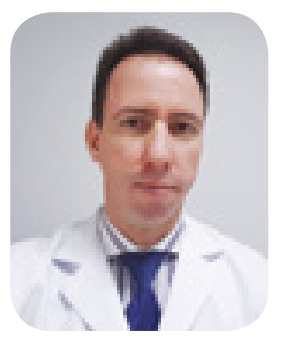

\author{
Gustavo Feitosa', Gilson Feitosa ${ }^{1}$
}

\begin{abstract}
Artigo original: Spironolactone Versus Clonidine as a Fourth-Drug Therapy for Resistant Hypertension The ReHOT Randomized Study (Resistant Hypertension Optimal Treatment) Autores: Eduardo M. Krieger, Luciano F. Drager, Dante M.A. Giorgi, Alexandre C. Pereira,José Augusto Soares Barreto-Filho, Armando R. Nogueira, José Geraldo Mill, Paulo A. Lotufo, Celso Amodeo, Marcelo C. Batista, Luiz C. Bodanese, Antônio C.C. Carvalho, Iran Castro, Hilton Chaves, Eduardo A.S. Costa, Gilson S. Feitosa, Roberto J.S. Franco, Flávio D. Fuchs, Armênio C. Guimarães, Paulo C. Jardim, Carlos A. Machado, Maria E. Magalhães, Décio Mion Jr., Raimundo M. Nascimento,Fernando Nobre, Antônio C. Nóbrega, Antônio L.P. Ribeiro, Carlos R. Rodrigues-Sobrinho,Antônio F. Sanjuliani,† Maria do Carmo B. Teixeira, José E. Krieger, ReHOTInvestigators. Publicado em Hypertension. 2018
\end{abstract} Apr;71(4):681-690

A hipertensão arterial resistente tem merecido interesse por conta da sua maior gravidade e da sua abordagem ainda não muito bem estabelecida..$^{1-3}$

Nosso estudo - The ReHOT Randomized Study (Resistant Hypertension Optimal Treatment) - foi realizado em portadores de hipertensão resistente, - definida como PA maior que 140/90mmHg em consultório, confirmada por PA maior que $130 / 80 \mathrm{mmHg}$, em média, na monitorização ambulatorial da pressão arterial (MAPA), após incrementos sucessivos na dosagem de 3 classes de anti-hipertensivos, obtendo-se o seguinte esquema posológico: clortalidona, $25 \mathrm{mg}$ ao dia; enalapril, $20 \mathrm{mg}, 2$ vezes ao dia (ou losartan $50 \mathrm{mg}, 2$ vezes ao dia); e amlodipina, $5 \mathrm{mg}$, 2 vezes ao dia.

Foram então randomizados para uso de clonidina $0,100 \mathrm{mg}, 2$ vezes ao dia, ou espironolactona, $12,5 \mathrm{mg}$ ao dia. Tais doses eram aumentadas para clonidina, 0,20 a 0,30 mg, 2 vezes ao dia, ou espironolactona 25 a $50 \mathrm{mg}$, com o intuito de atingir o controle da pressão arterial.

O desfecho primário foi o controle adequado da PA em 12 semanas de acompanhamento.

Os desfechos secundários foram o controle de PA por cada método de avaliação, consultório ou ambulatorial e absoluta redução de PA em cada grupo.

O estudo foi realizado em 26 centros brasileiros de cardiologia e os investigadores do Hospital Santa Izabel participaram do delineamento do estudo e de sua execução.

O estudo foi conduzido de outubro de 2010 a fevereiro de 2014 e foram incluídos para avaliação inicial 1.597 pacientes, sendo que ao cabo de cuidadosa investigação verificou-se uma verdadeira hipertensão resistente em 187 (11,7\%). Comparando-se inicialmente os verdadeiramente resistentes $(n=187)$ com os não resistentes $(n=1.410)$, verifica-se maior prevalência de dislipidemia, diabetes mellitus, história prévia de acidente vascular cerebral e menor taxa de filtração glomerular.

Também as cifras pressóricas de consultório ou na MAPA sempre foram maiores nos verdadeiramente resistentes.

Os pacientes randomizados para uso da clonidina ou espironolactona apresentavam características clínico-demográficas semelhantes, incluindo o escore de Berlim para apneia obstrutiva do sono, que estava presente como indicativo de alto risco em $65,2 \%$ no grupo de clonidina e $57,4 \%$ no grupo da espironolactona.

A figura 1 representa o comportamento das pressões sistólica e diastólica nos dois grupos em medidas de consultório, verificando-se resultados semeIhantes entre os 2 grupos.

Da mesma forma, na figura 2, verifica-se resultados semelhantes em medidas ambulatoriais da pressão arterial.

A despeito disso, o manuseio das doses foi melhor obtido com a espironolactona do que com a clonidina, por conta de efeitos colaterais mais associados a esta última, como sonolência. Por este motivo, na nossa visão, a espironolactona é, como quarto anti-hipertensivo, a medicação preferível para o controle de hipertensão arterial resistente.

Ademais, há expectativa a respeito de se caracte- 
rísticas fenotípicas seriam capazes de predizer meIhor resposta à clonidina ou espironolactona, dado o fato de ter sido caracterizada a atividade do sistema renina-angiotensina aldosterona (através de dosagem sérica e urinária) e atividade simpática através da variabilidade da FC em ECG de 20 minutos. Essa análise deverá ser realizada de forma retrospectiva e publicada em breve.

Este trabalho se destaca no cenário da hipertensão arterial resistente, por ser metodologicamente muito rigoroso e ser uma importante contribuição brasileira ao tema de grande relevância internacional.

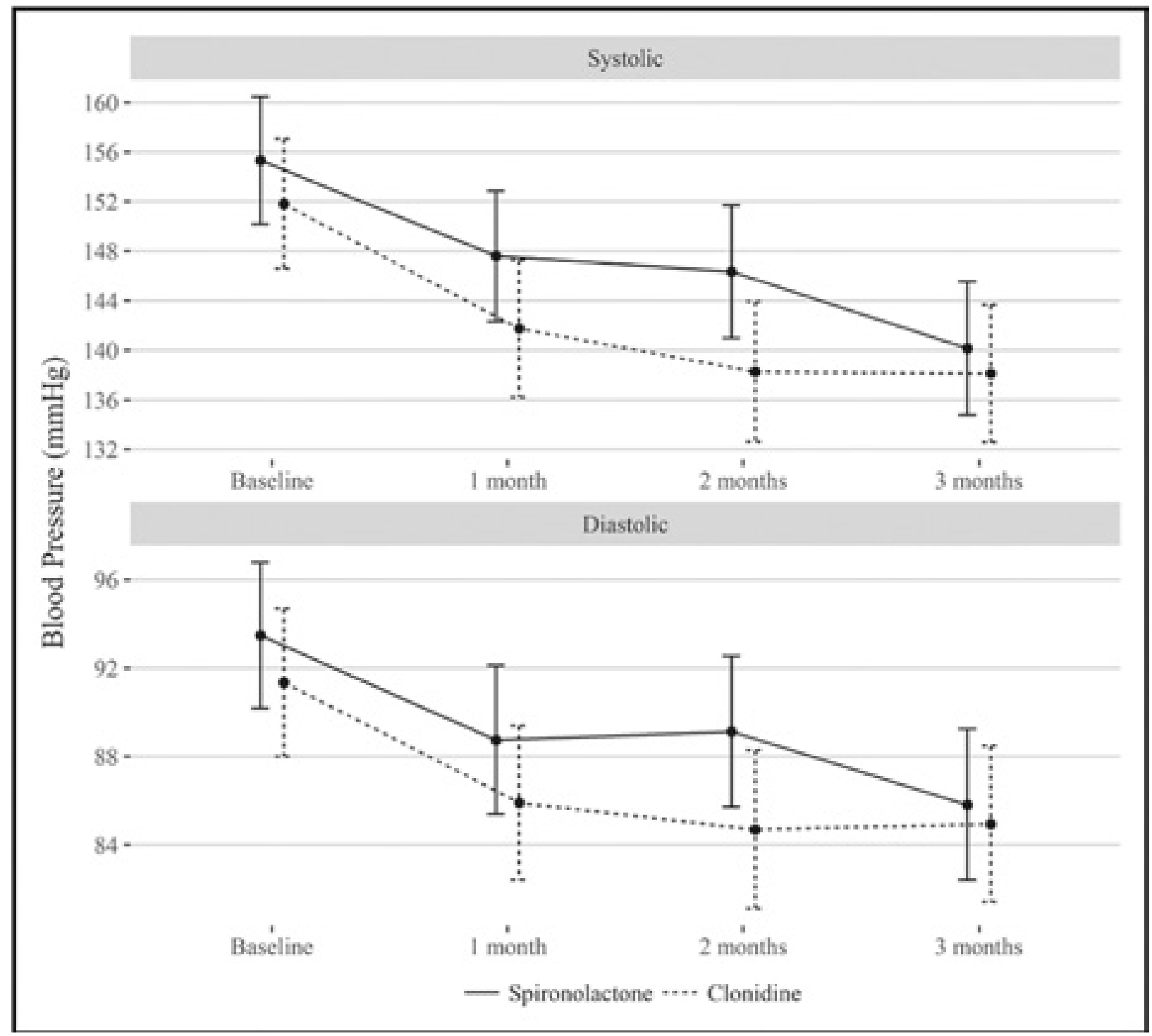

Figura 1. Adaptada da publicação original: Hypertension. 2018 Apr;71(4):681-690. 


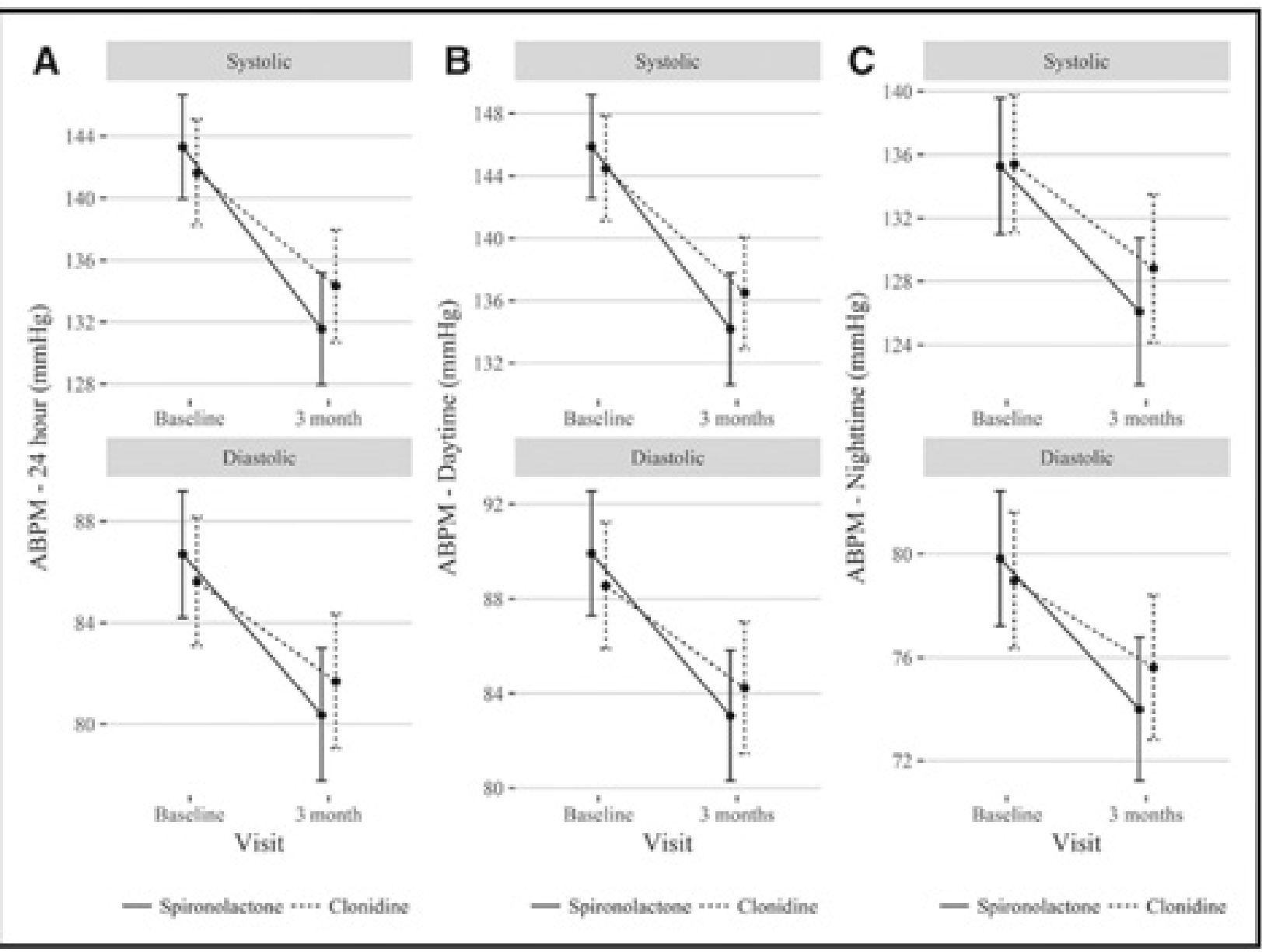

Figura 2. Adaptada da publicação original: Hypertension. 2018 Apr;71(4):681-690

\section{REFERÊNCIAS}

1- Calhoun DA, Jones D, Textor S, Goff DC, Murphy TP, et al . Resistant hypertension: diagnosis, evaluation, and treatment.A scientific statement from the American Heart Association Professional Education Committee of the Council for High Blood Pressure Research. Hypertension. 2008;51:1403-1419.

2- Williams B, MacDonald TM, Morant S, et al and British Hypertension Society's

PATHWAY Studies Group. Spironolactone versus placebo, bisoprolol, and doxazosin to determine the optimal treatment for drug-resistant hypertension (PATHWAY-2): a randomised, doubleblind, crossover trial. Lancet. 2015;386:2059-2068.
3- Lotufo PA, Pereira AC, Vasconcellos PS, Santos IS, Mill JG, Bensenor IM. Resistant hypertension: risk factors, subclinical atherosclerosis, and comorbidities among adults-the Brazilian Longitudinal Study of Adult Health (ELSA-Brasil). J Clin Hypertension 2015;17:74-80.

1- Serviço de Cardiologia do HSI Endereço para correspondência: gustavoffeitosa@yahoo.com.br 